\title{
Clinical Experience Using the SRS MapCHECK for Patient Specific Plan Verification of Flattening Filter Free, Non-Coplanar Stereotactic Brain Plans Using HyperARC.
}

Mark Ashburner ( $\square$ ashburnermark@gmail.com )

Waikato District Health Board

Koki Mugabe

Waikato District Health Board

\section{Research Article}

Keywords: FFF, SRS Brain, HyperARC, SRS MapCHECK

Posted Date: June 2nd, 2021

DOI: https://doi.org/10.21203/rs.3.rs-570978/v1

License: (c) (1) This work is licensed under a Creative Commons Attribution 4.0 International License.

Read Full License 


\section{Abstract}

HyperArc (HA) treatment planning from Varian is a stereotactic specific planning tool enabling quick and efficient optimisation of treatment planning, and delivery. HA was commissioned and implemented at Waikato Regional Cancer Centre (WRCC) in 2019 to fulfil the demands of dose delivery for stereotactic radiosurgery (SRS), allowing for treatment of multiple targets with a single isocenter at non-coplanar angles. The extra levels of plan complexity involved in creating and verifying HA SRS plans required extensive checks and verifications using film and an ion chamber, along with a significant allocation of time and resources. The Sun Nuclear SRS MapCHECK (SRSMC) offered an alternative to the cumbersome film measurements. It is an all-encompassing tool meeting the requirements of TG 218 and ICRU 91 for complex treatment plan verification, claiming to save time and effort, without sacrificing accuracy, enabling for a smoother planning and verification process.

SRSMC was initially commissioned on $6 \mathrm{MV}$ single target treatments using standard planning, then updated and commissioned for 6FFF multi-target non-coplanar treatments using HA.

The SRSMC gamma pass rates were compared to film measurements in the same plane, and the central diode CAX reading compared to ionisation chamber measurements at the same position for a range of plans covering a range of PTV sizes and plan complexities.

Pass rates on the SRSMC were comparable to measurements using film (Gamma 3\%/1 mm, 99.41\%, $96.39 \%$ SRSMC and film respectively). The central diode is an adequate surrogate for a chamber measurement if the SRSMC is positioned in a similar position as that of the ionisation chamber would be - high dose homogenous region, avoiding steep gradients (mean dose difference Diode vs Chamber: $-0.73 \%$ ). Differences between exposing non-coplanar plans at couch 0 and at planned couch angles were negligible (Gamma 3\%/1 mm 99.28 coplanar, 99.41\% non-coplanar on SRSMC).

At WRCC the SRSMC has replaced film and chamber measurements for plan verifications of 6FFF HA multiple metastatic brain treatments at a single isocenter and we are currently investigating its use in other treatment sites.

\section{Introduction}

Stereotactic radiosurgery (SRS) is recommended as the standard of care by the American Society of Radiation Oncology (ASTRO) (1) and the International Stereotactic Radiosurgery Society (ISRS) (2) for the primary treatment of patients with limited or multiple brain metastases and as an alternative to postoperative whole brain radiotherapy (3). The option to treat patients using SRS techniques has been available at Waikato Regional Cancer Centre (WRCC) since early 2017, and is now the standard of care for treatment of multiple metastatic brain lesions.

HyperArc (HA) (HyperArc ${ }^{T M}$, Varian Inc.) addresses the problems associated with resources allocation, and plan complexity when it comes to planning optimal treatments for multiple metastatic brain lesions. HA is designed to simplify and automate non-coplanar SRS planning for multiple-lesions at a single 
treatment position, negating the need for repositioning of the patient. This saves time when compared to treatments that treat multiple targets as separate plans, where each lesion has its own isocentre. WRCC introduced HA in 2019 to meet the demand for increasing cases and to provide the recommended international standard of care for patient's requiring radiation treatment for multiple metastatic brain cancers. HA plans have been shown to perform better than multi-isocenter VMAT plans in terms of both dose-volume quality metrics and overall treatment times, being completed within a typical time slot means that HA offers an easy and safe alternative to multi-isocenter VMAT for complex SRS for multiple metastases (4).

Typically patient-specific QA for SRS plans include verification of patient setup/ immobilization and dose delivery measurements when appropriate, and a dry-run of the approved treatment plan to check for potential collision. Where MLCs modulate the dose, measurements should be performed prior to treatment to verify the absolute dose to the reference point (5). Initially these measurements were done through the use of an in-house phantom utilising film and ionisation chamber measurements. Although EBT-XD films are well suited to achieve optimal results in SRS and SBRT plan verification(6) the process can be cumbersome in terms of accuracte set-up, calibration and orientation. These issues, coupled with a waiting time of at least 6 hours between exposure and analysis(7) leads to a significant time and resource allocation increasing pressure on the medical physics team, to accurately deliver and verify patient specific QA measurements on time.

The Sun Nuclear SRS MapCHECK (SRSMC) is an all-encompassing tool verified as a suitable surrogate for film(8) (9), which meets the requirements of AAPM TG 101(10) and ICRU report 91(11) for complex treatment plan verification whilst claiming to save time and effort, without sacrificing accuracy, overall enabling a smoother planning and verification process. The device consists of $1013 \mathrm{n}$-type solid state (Sunpoint ${ }^{\circledR} 2$ 2) diode detectors arranged in a $77 \mathrm{~mm}^{2}$ array in polymethyl methacrylate (PMMA), spacing between detectors is $2.47 \mathrm{~mm}$ with an active volume of $0.007 \mathrm{~mm}^{3}$, active detector area is $0.48 \times 0.48 \mathrm{~mm}^{2}$ and $0.5 \mathrm{~mm}$ resolution. Four fiducials which are visible both on $\mathrm{kV}$ and $\mathrm{MV}$ imaging along with external contours of the accompanying StereoPHAN ${ }^{\mathrm{T}}$, allow for accurate alignment for pre-treatment verification measurements

The StereoPHAN is a specific phantom designed to be compatible with SRSMC. For verification it is locked to the treatment couch preventing accidental motion, which in turn allows for the different dosimetry inserts to be used without adjusting the placement of the StereoPHAN on the couch. This enables comparisons between different measurement mediums without introduction of positioning uncertainty beyond those intrinsic to the system (12). For this work, the film holder, SRSMC and Microchamber insert were interchanged within the StereoPHAN.

Detailed is the experience of WRCC of using the SRSMC for $6 \mathrm{MV}$ with flattening filter (6WFF) and 6MV flattening filter free (6FFF) HA treatment plan verifications using both planar and non-coplanar field arrangements. 


\section{Methods}

SRT plan verification was historically conducted using a combination of ion-chamber point dose measurements (CC01) and film measurements (Gafchromic ${ }^{\text {TM }}$ EBT-XD, ESPON Expression 1100XL) in a bespoke head and neck phantom (originally a CIRS Model 002HN IMRT Phantom, modified for stereotactic purposes). Gamma analysis was carried out using OmniPro-ImRT (v1.6) film analysis software. Commissioning of SRSMC was done by verifying diode responses by looking at linearity, short/ long term reproducibility, obliquity and determination of calibration factors for absolute dose measurements. Absolute dose consisted of collecting an SRMC measurement under conditions in which the dose to the central detector is known, for entering into the SNC software, which correlates the counts collected by the device during calibration to the known dose entered by the user, which established the absolute dose calibration factor. The accuracy was tested by changing the dose delivered and comparing the dose change on the CAX measurement. The commissioning didn't reveal anything that would raise concerns in terms of clinical use, and showed similar results to the works carried out elsewhere (8) (9) (12). Results are not discussed in detail in this report as the authors did not want to present the work as a commissioning report, but rather wanted to describe the ongoing process of moving to a new planning technique and the opportunities that arose in terms of comparisons that can be carried out to further test the flexibility of the SRSMC for different types of stereotactic planning techniques. Once the array was determined to be acceptable for clinical measurements, a retrospective re-verification of previous SRS patient plans was carried out to determine whether or not there was significant differences in gamma pass rates when using our old verification methods compared to the new SRSMC. During this phase of commissioning non-coplanar plans were reset as coplanar as there was concerns about the risks involved through possible irradiation of the device electronics. In 2020 SRSMC was recommissioned for non-coplanar 6FFF plans using HA techniques, and full non co-planar geometry was included in verifications on the recommendations of the vendor, details and results of which are presented. In all cases the gamma index pass rates at WRCC are $95 \%$ of points pass at $3 \% 1 \mathrm{~mm}$ or at $5 \% 1 \mathrm{~mm}$

\section{I: SRSMC vs Film:}

Previous film verification of brain SRT plans and absolute gamma analysis was carried out at noncoplanar treatment angles in a bespoke, modified CIRS head and neck phantom in the transverse plane. A calibration curve was produced for each verification exposure using film taken from the same batch and sheet exposed to known doses of radiation. The phantom was positioned using room lasers then image matched using СBCT and matched to isocenter. Lateral shifts could then be applied using couch controls where required, once positioning was verified the plan was exposed at non-coplanar angles.

Following full acceptance, commissioning and calibration of the SRSMC a cohort of patients were replanned on the SRSMC for verification and validation. The SRSMC does not allow for dose measurements to be done in the transverse plane, therefore it was placed in the StereoPHAN at 0区 (coronal plane), the set-up verified using CBCT matching and the plans subsequently exposed. This difference in exposure planes and couch angles meant that a direct comparison between verification 
methods could not be carried out at this stage, instead as part of commissioning we verified that we had no false positives and the gamma passing rates were similar.

The StereoPHAN comes with an insert for films, meaning verification plans could be repeated using film in the same plane and setup as the SRSMC, meaning a more meaningful comparison between film and SRSMC could be done. A cohort of plans covering all PTV sizes and non-coplanar angles were re-planned in the StereoPHAN using both the SRSMC and film insert, allowing for comparison in the same plane and phantom. A film calibration curve was done on each day of exposure from $2 \mathrm{~Gy}$ to $+10 \%$ max planned doses.

\section{II: CAX vs lonisation Chamber.}

The CAX Diode is located in the centre of the SRSMC, and is considered to be located at isocenter in isocentric set up, the software gives a value of absolute dose to this point in both the planned and exposed planes (labelled CAX2 and CAX1 respectively). Cumulative dose to both the ionisation chamber and CAX diode was directly compared at non-coplanar geometry. Off-axis shifts were applied to the SRSMC when required to ensure that the CAX diode was in the same position as the initial point of measurement of the chamber for the clinical plan, which was set up to be in a high dose, homogenous region of the PTV. SNC Patient displays the CAX diode dose for both the exposed and planned dose planes, so a direct comparison was carried out to see if dose differences between planned and exposed are similar to that of the TPS vs Exposed when done with an ion-chamber. Plans covered all non-coplanar types of measurement carried out at WDHB including 6WFF, 6FFF beams, and central and off axis measurements.

\section{III: 6WFF vs 6FFF:}

Separate and individual dose and array calibration files for both 6WFF and 6FFF energies are stored in SNC Patient software for use dependent on the energy used for the treatment plan. Practice evolved at WRCC from 6WFF to 6FFF for treatment of SRS Metastatic brain cancer. Part of the commissioning for this a selection of clinical SRS plans using 6WFF were re-planned with same beam parameters and setup, but beam energy changed to 6FFF. Both sets of plans were exposed on the SRSMC, at coplanar set up and gamma analysis pass rates compared.

\section{IV: Coplanar vs Non Coplanar.}

During the initial commissioning phase clinical plans covering all PTV sizes and couch angles were exposed with all fields set to coplanar angles. A cohort of these plans were re-planned and exposed at full non-coplanar geometry and gamma analysis conducted for comparison.

\section{V: Isocentric Vs off axis:}

Plan verification of multiple targets may require shifting the SRSMC away from isocenter to ensure all planned PTVs fit on the SRSMC detector panel. Once we moved to planning with HA a review a 
retrospective analysis of pass rates of plans done at isocentric set up and off axis were compared.

\section{Results \& Discussion}

\section{I: SRSMC vs Film}

As previously discussed, due to the composition of the bespoke head and neck phantom plan verifications were historically exposed on film in the transverse plane. Initial commissioning of the SRSMC was done with a coplanar set up meaning in the coronal plane, therefore it was not possible to have the diode and film exposures in the same plane for direct comparison.

Instead, for commissioning, we verified that pass rates were of a similar magnitude for the plan as a whole complex treatment, i.e. pass rates $>95 \%$ at $1 \% 2 \mathrm{~mm}$ gamma index would have similar pass rates in both the coronal and transverse planes, but would not expect them to be identical.

Average pass rates for a selection ( $\mathrm{N}=20)$ of original treatment plans were $91.05 \%$ at $3 \% 1 \mathrm{~mm}$ and $97.29 \%$ at $5 \% 1 \mathrm{~mm}$. The corresponding average pass rates of the same plans exposed at coplanar angles, on the SRSMC in the coronal plane were $99.41 \%$ and $99.9 \%$ for $3 \% 1 \mathrm{~mm}$ and $5 \% 1 \mathrm{~mm}$ respectively. A selection of these plans covering a range of PTV equivalent diameters are highlighted in Table 1 and average results over all plans summarised in Table 3.

Table 1

Original film plans in transverse plane Vs SRSMC at coplanar and non-coplanar couch angles in coronal plane.

\begin{tabular}{|c|c|c|c|c|c|c|}
\hline \multirow[t]{3}{*}{ PTV EQD (cm) } & \multirow{2}{*}{\multicolumn{2}{|c|}{ Film }} & \multicolumn{4}{|l|}{ SRSMC } \\
\hline & & & \multicolumn{2}{|l|}{ Coplanar } & \multicolumn{2}{|c|}{ Non Coplanar } \\
\hline & $3 \% / 1 \mathrm{~mm}$ & $5 \% / 1 \mathrm{~mm}$ & $3 \% / 1 \mathrm{~mm}$ & $5 \% / 1 \mathrm{~mm}$ & $3 \% / 1 \mathrm{~mm}$ & $5 \% / 1 \mathrm{~mm}$ \\
\hline 1.2 & 97.28 & 98.41 & 97.7 & 100 & 99.3 & 100 \\
\hline 1.4 & 83.18 & 96.08 & 99.6 & 100 & 100 & 100 \\
\hline 1.5 & 94.39 & 98 & 100 & 100 & 100 & 100 \\
\hline 2.4 & 94.06 & 95.94 & 99.6 & 100 & 94.7 & 99.2 \\
\hline 2.5 & 91.41 & 97.91 & 99.5 & 100 & 99.8 & 100 \\
\hline 2.6 & 91.81 & 96.96 & 100 & 100 & 99.7 & 100 \\
\hline 3.4 & 99.16 & 99.71 & 98.6 & 99.8 & 98.8 & 100 \\
\hline 4.3 & 91.73 & 99.26 & 99.6 & 100 & 100 & 100 \\
\hline
\end{tabular}

Differences in pass rates are possibly due to a number of reasons: 
- The original plans were conducted by several different Physicists over a period of a couple of years, meaning inherent variations in output on the day, localisation and user analysis are taken in to account over time, as opposed to the plans for this study which were conducted in single sessions, by a single Physicist.

- The resolution of the film vs the resolution of the SRSMC diode plane, whereby a higher resolution (film) is more likely to highlight smaller errors, which may not be picked up by the diodes leading to a lower gamma index pass rate.

- Differences in matching techniques due to the phantoms used. Both methods used CBCT preexposure image matching, however the homogenous SRSMC and StereoPHAN phantom has ball bearings embedded for localisation, along with other easily discernible physical features on the СBCT image. 6dof couch corrections were also available, which would not have been present for some of our previous film measurements.

The SRSMC allows exposure in the coronal or sagittal planes, and in order to have a like-for-like comparison, a selection of historic plans were re-exposed using the film holder in the StereoPHAN, then compared to measurements on the SRSMC in the same plane, the results of which are presented in Table 2 and Table 3.

Pass rates for the SRSMC were consistently higher than those obtained with film, being $100 \%$ pass rate at $3 \% 1 \mathrm{~mm}$ consistently. Differences between film and SRSMC pass rates varied, $0.16-9.37 \%$ difference at $3 \% / 1 \mathrm{mmv}$ (after re-measurement and verification). The possible reasons for these differences are discussed above. We would not consider the SRSMC results to be a false positive, however when using the device for plan verification users should be aware of these points when replacing film. 
Table 2

Direct comparison of film measurements and SRSMC in coronal plane in StereoPHAN phantom.

\begin{tabular}{|c|c|c|c|c|c|c|}
\hline \multirow[t]{2}{*}{ PTV Eq Di (cm) } & \multicolumn{2}{|l|}{ Film } & \multicolumn{2}{|l|}{ SRSMC } & \multicolumn{2}{|l|}{ Difference } \\
\hline & $3 \% / 1 \mathrm{~mm}$ & $5 \% / 1 \mathrm{~mm}$ & $3 \% / 1 \mathrm{~mm}$ & $5 \% / 1 \mathrm{~mm}$ & $3 \% / 1 \mathrm{~mm}$ & $5 \% / 1 \mathrm{~mm}$ \\
\hline 1.2 & 97.57 & 98.7 & 100 & 100 & 2.13 & 1.30 \\
\hline 1.6 & 99.84 & 100 & 100 & 100 & 0.16 & 0.00 \\
\hline 1.8 & 97.97 & 99.5 & 100 & 100 & 2.03 & 0.50 \\
\hline 1.9 & 93.88 & 95.5 & 100 & 100 & 6.12 & 4.50 \\
\hline 2.1 & 99.69 & 99.91 & 100 & 100 & 0.31 & 0.09 \\
\hline 2.2 & 96.5 & 99.2 & 100 & 100 & 3.50 & 0.80 \\
\hline 3.0 & 91.92 & 92.31 & 100 & 100 & 8.08 & 7.69 \\
\hline 3.2 & 99.51 & 97.84 & 100 & 100 & 0.49 & 2.16 \\
\hline 4.1 & 90.63 & 92.09 & 100 & 100 & 9.37 & 7.91 \\
\hline
\end{tabular}

Table 3

Summary of average results for film analysis vs SRSMC in various setups

\begin{tabular}{|c|c|c|c|c|c|c|c|}
\hline \multicolumn{2}{|c|}{ SRSMC Coplanar } & \multicolumn{2}{|c|}{ SRSMC Non-Coplanar } & \multicolumn{2}{|c|}{ Film: Custom Phantom } & \multicolumn{2}{|c|}{ Film: StereoPHAN } \\
\hline $3 \% / 1 \mathrm{~mm}$ & $5 \% / 1 \mathrm{~mm}$ & $3 \% / 1 \mathrm{~mm}$ & $5 \% / 1 \mathrm{~mm}$ & $3 \% / 1 \mathrm{~mm}$ & $5 \% / 1 \mathrm{~mm}$ & $3 \% / 1 \mathrm{~mm}$ & $5 \% / 1 \mathrm{~mm}$ \\
\hline 99.3 & 100 & 99.41 & 99.9 & 91.05 & 97.29 & 96.39 & 97.23 \\
\hline
\end{tabular}

\section{II: CAX Diode vs Ionisation Chamber.}

Measurement on the SRSMC at non-coplanar geometry allows direct comparison of the cumulative dose measured on the CAX Diode and with an ionisation chamber at the same position. Results showed good agreement between the ion chamber measurement and the central diode. It was noted that the results for the chamber are from historic measurements and retrospectively compared to recent CAX measurements done as part of this study. Similar issues as those discussed above, with different team members taking measurements and inherent daily machine variations were taken into consideration when interpreting the differences between the ion chamber and CAX diode, summarised as a selection of plans in Table 4 and average of all plans in Table 5.

The large discrepancy of $3.47 \%$ was attributed to a small volume PTV (1.2cm equivalent diameter). This also corresponded to $-4.43 \%$ difference seen in the CAX1 (measured) vs CAX2 (TPS) measurement. However, in this instance, the actual difference in diode vs ion chamber was $0.34 \%$. Although this was a 
large discrepancy for both the difference in diode and chamber measurement when compared to the TPS, the diode accurately reflects what is measured by the chamber, therefore we were satisfied that the CAX diode is a suitable surrogate for an ion-chamber to measure point dose.

Table 4

Selection of Ion Chamber and CAX Diode measurements. (* Highlighted and discussed result)

\begin{tabular}{|llll|}
\hline PTV Equivalent Diameter $(\mathbf{c m})$ & IC Vs TPS & CAX Diode Vs IC & CAX1 Vs CAX 2 \\
\hline $\mathbf{1 . 2}{ }^{*}$ & 3.47 & -0.34 & -4.43 \\
\hline $\mathbf{1 . 4}$ & 0.66 & -2.22 & 1.36 \\
\hline $\mathbf{1 . 4}$ & 1.06 & -1.69 & -1.68 \\
\hline $\mathbf{1 . 5}$ & 3.39 & -1.85 & -1.87 \\
\hline $\mathbf{1 . 8}$ & 1.88 & 0.66 & -1.74 \\
\hline $\mathbf{2 . 1}$ & -0.38 & -1.09 & -2.67 \\
\hline $\mathbf{2 . 4}$ & 1.21 & 0.62 & -3.84 \\
\hline $\mathbf{2 . 5}$ & -0.48 & -2.22 & -0.48 \\
\hline $\mathbf{3 . 4}$ & 1.47 & -0.55 & -3.85 \\
\hline $\mathbf{4 . 3}$ & 2.57 & -2.14 & 0.08 \\
\hline
\end{tabular}

Table 5

Summary of lon Chamber and CAX Dose Measurements.

\begin{tabular}{|llll|}
\hline Variables & $\begin{array}{l}\text { Mean Difference } \\
(\%)\end{array}$ & $\begin{array}{l}\text { Standard } \\
\text { Deviation }\end{array}$ & $\begin{array}{l}\text { Range of Differences } \\
\text { (\%) }\end{array}$ \\
\hline $\begin{array}{l}\text { lon Chamber TPS Dose vs Exposed } \\
\text { Dose }\end{array}$ & 0.72 & 1.5 & -2.49 to 3.47 \\
\hline lon Chamber vs CAX Diode Dose & -0.48 & 1.35 & -2.57 to 0.84 \\
\hline CAX 1 vs CAX 2 Dose & -0.91 & 1.51 & -4.43 to 1.92 \\
\hline
\end{tabular}

\section{III: 6FFF vs 6WFF.}

Exposures used at commissioning of 6FFF $(\mathrm{N}=10)$ were utilised here to investigate the SRSMC gamma pass rates when the same plan is exposed with and without a flattening filter. The differences in pass rates were negligible, and the average gamma analysis pass rates and differences are presented in Table 6. 
Table 6

Summary of average gamma pass rates and average differences for treatments using 6 WFF and 6FFF

\begin{tabular}{|lllllll|}
\hline & 6WFF & & 6FFF & \multicolumn{3}{c|}{ Difference (\%) } \\
\hline & $\mathbf{3 \%} \mathbf{1 m m}$ & $\mathbf{5 \%} \mathbf{1 m m}$ & $\mathbf{3 \%} \mathbf{1 m m}$ & $\mathbf{5 \%} \mathbf{1 m m}$ & $\mathbf{3 \%} \mathbf{1 m m}$ & $\mathbf{5 \%} \mathbf{1 m m}$ \\
\hline Ave & 99.4 & 100.0 & 99.0 & 99.9 & 0.4 & 0.1 \\
\hline SD & 1.0 & 0.1 & 1.0 & 0.3 & 1.3 & 0.3 \\
\hline Min & 96.9 & 99.7 & 97.2 & 98.9 & -2.3 & -0.3 \\
\hline Max & 100.0 & 100.0 & 100.0 & 100.0 & 2.8 & 1.1 \\
\hline
\end{tabular}

\section{IV: Co-planar vs non-coplanar.}

A different cohort of clinical plans used for commissioning initially exposed with all fields to be coplanar were repeated using couch angles determined by HA planning techniques $(\mathrm{N}=20)$. Plans analysed were selected to cover all PTV sizes and non-coplanar angles. The average of the pass rates for coplanar and non-coplanar are summarised in Table 7.

Table 7

Summary of average pass rates for the same plans done at coplanar and non-coplanar angles

\begin{tabular}{|llll|}
\hline Coplanar & & \multicolumn{2}{l|}{ Non-Coplanar } \\
\hline $\mathbf{3 \%} \mathbf{1 m m}$ & $\mathbf{5 \%} \mathbf{1 m m}$ & $\mathbf{3 \%} \mathbf{1 m m}$ & $\mathbf{5 \%} \mathbf{1 m m}$ \\
\hline 99.28 & 99.95 & 99.41 & 99.90 \\
\hline
\end{tabular}

No significant difference in results were noted when the same plan is exposed at couch 0 and when the couch is rotated.

\section{V: Isocentric vs Off-axis}

A retrospective review and comparison of the pass rates of plans done at isocentric set up and off axis covering all target sizes and lateral shifts were compared. A selection are presented in Table 8 , and summarised in Table 9. 
Table 8

Pass rates for selection of plans at both Isocentric and off axis locations.

\begin{tabular}{|llll|}
\hline \multirow{4}{*}{ Isocentric } & \multicolumn{1}{l}{ PTV Eq Di $(\mathrm{cm})$} & $3 \% 1 \mathrm{~mm}$ & $5 \% 1 \mathrm{~mm}$ \\
\cline { 2 - 4 } & 1.2 & 99.7 & 100 \\
\cline { 2 - 4 } & 2.1 & 100 & 100 \\
\cline { 2 - 4 } & 2.6 & 99.9 & 100 \\
\hline \multirow{3}{*}{ Off-Axis } & 3.0 & 100 & 100 \\
\cline { 2 - 4 } & 1.3 & 100 & 100 \\
\cline { 2 - 4 } & 1.4 & 96.1 & 97.7 \\
& 1.6 & 99.3 & 100 \\
\cline { 2 - 4 } & 2.2 & 99.3 & 99.9 \\
\hline & 2.6 & 98.1 & 100 \\
\hline
\end{tabular}

Table 9

Summary of average pass rates for isocentric and off-axis plans

\begin{tabular}{|llll|}
\hline Iso & \multicolumn{3}{|c|}{ Off Axis } \\
\hline $\mathbf{3} \% \mathbf{1 m m}$ & $\mathbf{5} \% \mathbf{1 m m}$ & $\mathbf{3} \% \mathbf{1 m m}$ & $\mathbf{5 \%} \mathbf{1 m m}$ \\
\hline 99.58 & 100.00 & 97.86 & 99.66 \\
\hline
\end{tabular}

As both sets of results give a clinical pass, the slight difference in results was deemed to be of no concern. The $3 \% 1 \mathrm{~mm}$ off-axis results tended to have a lower passing rate when exposed at non-coplanar angles, likely due to inclusion of lateral couch movements introducing uncertainties which are reflected in the final measurement. However, these were still comfortably within the acceptable limits and tolerances, but the lower pass rate fortifies the need for an isocenter verification test, such as the Winston-Lutz test, to be carried out routinely as part of a SRT program. At WRCC this is checked weekly.

\section{Discussion}

Presented is the clinical experience of our centre of moving from a more traditional film and chamber measurement to diodes using SRSMC for SRS Brain metastases plan verification. As we progressed to using $6 \mathrm{FFF}$ in place of $6 \mathrm{WFF}$, and adapted $\mathrm{HA}$ treatment planning techniques we were able to utilise more of the features available in the SNC software, being the CAX dose measurement and exposures at non- 
coplanar geometry and the authors were presented with the opportunity to make some direct comparisons for different types of stereotactic techniques, as we moved from single target planning using $6 \mathrm{WFF}$, and carrying out verifications at coplanar angles, to more advanced planning techniques of multi-target single-isocentre using 6FFF with full non-coplanar geometry verification.

There are no differences that would be of a clinical concern between film and SRSMC when exposed in the StereoPHAN in the same plane. Similarly the insignificance in differences between 6WFF and 6FFF coupled with similar results seen when comparing coplanar with non-coplanar means that the SRSMC is suitable for measurement and verification of treatments planned with HA and 6FFF.

The slight difference in pass rates when exposing plans off-axis may be indicative of the inherent errors when rotating and shifting the couch to get to the required position for exposure and should be considered when commissioning such a system, and further highlights the need to verify machine isocenter for stereotactic treatments.

The central axis diode is comparable in accuracy to the farmer chamber when comparing to planned dose.

\section{Conclusion}

Presented are the results of testing done at WRCC as part of initial clinical testing of the SRSMC and subsequent process changes when moving to planning multiple target brain metastases using SRS HA planning and 6FFF treatment techniques.

Gamma pass rates on the SRSMC are comparable to measurements done using film. The central diode is an adequate surrogate for a chamber measurement if the SRSMC is positioned in a similar position as that of the ionisation chamber, being a high dose homogenous region, avoiding steep gradients. Differences between exposing non-coplanar plans at couch 0 and at planned angles were negligible.

At WRCC the SRSMC has replaced film and chamber measurements for plan verifications of 6FFF multiple metastatic brain lesions at a single isocenter, significantly reducing the resource allocation for SRT metastatic brain plan checks, giving us significant savings in terms of time costs and resource allocation. Investigations are ongoing for its use in other treatment sites using stereotactic techniques including lung, spine and kidney.

\section{Declarations}

Conflicts of interest/Competing interests work was carried out with cooperation from Sun Nuclear and AlphaXRT

Ethics approval: Not needed for this work

Consent for publication Both authors consent for this work to be published under their names. 


\section{References}

1. Radiotherapeutic and surgical management for newly diagnosed brain metastasis(es): An American Society for Radiation Oncology evidence- based guideline. Tsao, May , et al. 2012, Practical Radiation Oncology, pp. 210-225.

2. Stereotactic Radiosurgery in the Management of Limited (1-4) Brain Metasteses: Systematic Review and International Stereotactic Radiosurgery Society Practice Guideline. Chao, Samuel, et al. 2018, Neurosurgery, pp. 345-353.

3. Suh, John, et al. Current approaches to the management of brain metastases. Nature Reviews Clinical Oncology. 20 feb 2020, pp. 279-300.

4. Linac Based VMAT Radiosurgery for multiple brain lesions: comparison bewteen a conventional multi-isocenter approach and a new dedicated mono-isocenter technique. Ruggieri, Ruggero, et al. 2018, Radiation Oncology, pp. 13-38.

5. AAPM-RSS Medical Physics Practice Guideline 9.a for SRS-SBRT. Halvorsen, Per, et al. 2017, Journal Applied Clinical Medical Physics, pp. 10-21.

6. Technical Note: Initial Charaterization of the new EBT-XD Gafchromic Film. Grams, Michael, et al. 2015, Medical Physics, pp. 312-322.

7. GafChromic EBT-XD film: Dosimetry charaterization in high-dose, volumetric-modulated arc therapy. Miura, Hideharu, et al. 2016, Journal Applied Clinical Medical Physics, pp. 312-322.

8. Comprehensive evaluation of teh high-resolution diode array for SRS dosimetry. Ahmed, Saeed, et al. 2019, Journal of Applied Clinical Medical Physics, pp. 1-11.

9. Initial Evaluation of a 2D Diode Array for Quality Assurance of Radiosurgery Treatments. RonquilloGomez, Roberto Carlos, et al. 2019. AIP Conference Proceedings 2090 020006. pp. 0200061-7.

10. Sterotactic body radiation therapy: The report of AAPM Task Group 101. Benedict, Stanley, et al. 8 , 2010, Medical Physics, Vol. 37, pp. 1-12.

11. Prescribing, Recording, and Reporting of Stereotactic Treatements with Small Photon Beams. s.I. : International Commision of Radiation Units and Measurments, 2017.

12. Multi-institution validation of a new high spatial resolution diode array for SRS and SBRT plan pretreatment quality assurance. Rose, Mark, et al. 7, July 2020, Medical Physics, Vol. 47, pp. 31533163. 